Supporting Information for

\title{
Role of Surface Templating on Ice Nucleation Efficiency on a Silver Iodide Surface
}

Zhuo Liu ${ }^{1,2}$, Chu $\mathrm{Li}^{1}$, Eshani C. Goonetilleke ${ }^{1}$, Yi Cui ${ }^{1}$, and Xuhui Huang ${ }^{1, *}$

${ }^{1}$ Department of Chemistry, Center of Systems Biology and Human Health, State Key

Laboratory of Molecular Neuroscience, The Hong Kong University of Science and

Technology, Kowloon, Hong Kong

${ }^{2}$ Institute for Advanced Study, The Hong Kong University of Science and Technology, Clear

Water Bay, Kowloon, Hong Kong

* To whom correspondence should be addressed. Email: xuhuihuang@ust.hk 


\section{Impact of system setups on water orientations and ice nucleation efficiency}

Sayer and Cox recently proposed a method to stabilize the AgI crystal by imposing an electric filed or electric displacement filed since the $\beta-\mathrm{AgI}(0001)$ surface is inherently unstable unless a polarity compensation mechanism prevails. ${ }^{1}$ We have performed additional MD simulations using two setups recommended in their work (i.e., $\boldsymbol{D}_{\mathbf{C N C}}$ and $\boldsymbol{D}_{\mathbf{C N C}}+\mathrm{NaCl}$ electrolyte, see SI Section 2 for the details of simulation setups). For the water orientations in the contact layer, we obtained consistent results as suggested by Sayer and Cox. ${ }^{1}$ In particular, the probability that the $\mathrm{O}-\mathrm{H}$ bonds in water molecules point toward or away from the $\beta$ - $\mathrm{AgI}(0001)$ surface in the mirrored slab geometry are comparable (Figure S1a), while the O-H bonds in water molecules preferentially point away from the surface in the polarity compensation setups $\left(\boldsymbol{D}_{\mathbf{C N C}}\right.$ in Figure S1c and $\boldsymbol{D}_{\mathbf{C N C}}+\mathrm{NaCl}$ electrolyte in Figure S1d).

To verify the distinct water orientations obtained from these different setups, which apply 3D-Ewald to the two-dimensional interfacial system, 2D-Ewald can be used as an ideal reference method; however, its direct application to our system remains too expensive. ${ }^{2}$ Therefore, we adopted a setup that has been previously reported to mimic the 2D-Ewald in producing both consistent water orientations and the force distribution perpendicular to the surface. ${ }^{3}$ In this setup, the Yeh and Berkowitz (YB) correction is used, but a large volume factor of 5 is chosen (i.e., the vacuum along the $z$-dimension of the simulation box is 4 times as large as that of the AgI surface plus all the water molecules, see Ref. 3). We show that MD simulations using this setup (YB with a volume factor of 5, see SI Section 2 for details of simulation setup) yield water orientations in the contact layer that are consistent with those obtained from the mirrored slab geometry (Figure S1a-b). We also note that several recent studies have successfully applied the mirrored slab geometry to correctly predict water orientations in contact with polar surfaces. For example, both Sum Frequency Generation spectroscopy ${ }^{4}$ and simulations using the mirrored slab geometry ${ }^{5}$ show that the net orientation 
of the $\mathrm{O}-\mathrm{H}$ bond in water points toward the $\mathrm{OH}$-terminated $\alpha-\mathrm{Al}_{2} \mathrm{O}_{3}(0001)$ surface. In another example, MD simulations using the mirrored slab geometry showed that the $\mathrm{O}-\mathrm{H}$ bonds of water molecules in the contact layer can be directed toward or away from the Al-terminated $\alpha-\mathrm{Al}_{2} \mathrm{O}_{3}(0001)$ surface, ${ }^{5}$ which is consistent with the DFT calculations. ${ }^{6}$ For $\boldsymbol{D}_{\mathbf{C N C}}$, the introduction of the electric displacement field can help to greatly stabilize the AgI crystal (we achieve this issue by fixing the AgI surface in our MD simulations). However, we anticipate that the presence of the electric field could also potentially impact the orientation of water molecules in the contact layer, as both previous experiment ${ }^{7}$ and simulation ${ }^{8}$ have shown that water molecules would reorient under an electric field. Nevertheless, we acknowledge that due to the lack of experimental validations, it would be difficult to draw affirmative conclusions on water orientations in the contact layer on the AgI surface.

More importantly, we find that our major conclusion is robust regardless of the electrostatic boundary conditions: i.e., the trend in templating effect for different AgI surfaces on the ice nucleation rate still holds using the mirrored slab geometry, the $\boldsymbol{D}_{\mathbf{C N C}}$, and the $\boldsymbol{D}_{\mathbf{C N C}}+\mathrm{NaCl}$ electrolyte (see Figure S2). We anticipate that it is the relative difference in water orientation distribution between the contact layer (before ice nucleation) and bulk ice, rather than the absolute water orientations in the contact layer itself, that determines the relative ice nucleation efficiency on various AgI surfaces.

In particular, we have performed 10 independent MD simulations for each of the two systems with different lattice mismatch $(\delta=0 \%$ and $\delta=1.59 \%)$ by following a previous protocol (see Ref. 1) to set up the $\boldsymbol{D}_{\mathbf{C N C}}$ and the $\boldsymbol{D}_{\mathbf{C N C}}+\mathrm{NaCl}$ electrolyte models. Using these two setups, we found that the ice nucleation rate when $\delta=0 \%$ (perfect lattice match) is substantially faster than that when $\delta=1.59 \%$ at a specific setup (Figure S2b-c). Interestingly, we found that water orientations in the contact layer when $\delta=0 \%$ display a closer distribution to bulk ice than those when $\delta=1.59 \%$ (Figure S2e-f), which may subsequently promote ice 
nucleation. These results obtained using the $\boldsymbol{D}_{\mathbf{C N C}}+\mathrm{NaCl}$ electrolyte setup are consistent with those from the mirrored slab geometry as well as the $\boldsymbol{D}_{\mathbf{C N C}}$ setup (Figure S2d-f). Even though we observed that perfect lattice match promotes ice nucleation in all three setups, we do acknowledge that the orientation of water molecules in the contact layer and the absolute ice nucleation rates can vary substantially across different system setups (see the insets of Figure S2a-c). For example, the $\boldsymbol{D}_{\mathbf{C N C}}+\mathrm{NaCl}$ electrolyte setup displays a slower ice nucleation rate than that of $\boldsymbol{D}_{\mathbf{C N C}}$, which may be due to the inhibiting effect of the adsorbed ions.

\section{System setups and simulation details}

To investigate the water orientations in the contact layer of AgI, we used the YB correction with a volume factor of 5. One single AgI slab contained four AgI layers with lattice parameters of $\mathrm{a}=4.590 \AA$ and $\mathrm{c}=7.512 \AA$. The lateral dimensions of the simulation cell were $\mathrm{Lx}=55.65$ $\AA$ and Ly $=55.08 \AA$. The $z$ dimension was $94.34 \AA$ with a single AgI slab in the center of our simulation box. For the YB correction, there is no periodic boundary condition along the $z$ direction, so the PPPM solver was modified to account for the slab geometry using the LAMMPS option based on the method of Yeh and Berkowitz with a volume factor of $5 .{ }^{3,9} \mathrm{We}$ added 2305 water molecules at the top and bottom of the simulation box, respectively. For the simulation performed at $298 \mathrm{~K}$, at least 100 ps of equilibration was conducted, followed by a further $2.0 \mathrm{~ns}$ of production. Other simulation protocols are the same as those for the mirrored slab geometry.

To verify the water orientations in the contact layer on $A_{g I}$ at $D_{\mathbf{C N C}}$, we followed the simulation protocol in Ref. 1. In particular, we used the TIP4P/2005 water model for the simulations, ${ }^{10}$ while the Madrid model ${ }^{11}$ was adopted for simulations involving sodium chloride. For the TIP4P/2005 model, each oxygen atom carried a charge of $-1.1128 \mathrm{e}$, and each hydrogen atom carried a charge of $0.5564 \mathrm{e}$. On the surface of AgI, silver and iodide ions were 
positively and negatively charged to $0.5815 \mathrm{e}$, respectively, and sodium and chloride ions carried a charge of $0.85 \mathrm{e}$ and $-0.85 \mathrm{e}$, respectively. The LJ parameters with a $10.0 \AA$ cut-off distance were taken from a previous study done by Hale and Kiefer, ${ }^{12}$ excluding the polarization contributions. ${ }^{13}$ In addition, the Lorentz-Berthelot mixing rules were applied to obtain non-electrostatic interactions between $\mathrm{NaCl}$ and $\mathrm{AgI}$. Parameters for non-electrostatic interactions are summarized in Table S1.

To study water orientations at $\boldsymbol{D}_{\mathbf{C N C}}$, we used lattice constants of a $=4.592 \AA$ and $\mathrm{c}=$ $7.510 \AA$ for our simulations. The AgI slab contained nine layers with $16 \mathrm{Ag}^{+}$and nine layers with $16 \mathrm{I}^{-}$. The slab width was $30.934 \AA$, and the lateral dimensions of the simulation cell were $\mathrm{Lx}=18.368 \AA$ and $\mathrm{Ly}=15.907 \AA$. The total length of the simulation box was $117.475 \AA$ along the $z$-direction, which we took to be normal to the AgI surface (based on the simulation set up in Ref. 1). We then filled the remaining volume (not occupied by AgI) with 750 water molecules. For simulations at $\boldsymbol{D}_{\mathbf{C N C}}+\mathrm{NaCl}$ electrolyte, three $\mathrm{NaCl}$ ion pairs were added into the water, which is the same as the setup in Ref. 1.

All MD simulations in this work were carried out in the $N V T$ ensemble using the LAMMPS simulation package; ${ }^{14}$ and all the surfaces were modelled as rigid at their lattice sites during our simulations. The constant electric displacement field was implemented by using the method in Ref. 15. The $\boldsymbol{D}_{\mathbf{C N C}}$ was $-14.99 \mathrm{~V} / \mathrm{nm}$ for $\mathrm{AgI}$ with a lattice parameter of a $=4.592 \AA$. The RATTLE algorithm was applied to maintain the rigid geometry of the TIP4P/2005 water molecules, ${ }^{16}$ and the temperature was maintained by the Nosé-Hoover thermostat with a relaxation time of $0.1 \mathrm{ps}$. The Coulombic interactions were cut off at $8.5 \AA$ with its long-range interactions computed by Particle-Particle Particle-Mesh (PPPM). ${ }^{17}$ For simulations performed at $298 \mathrm{~K}$, at least 100 ps of equilibration was performed, followed by a further 2.0 ns of production. Then, 2 ns simulations at $\boldsymbol{D}_{\mathbf{C N C}}$ and $\boldsymbol{D}_{\mathbf{C N C}}+\mathrm{NaCl}$ electrolyte were obtained to analyze the water orientations on AgI under an electric displacement field. To quantify the 
water orientations in the contact layer, we calculated the probability distributions of the $\mathrm{O}-\mathrm{H}$ bonds of water molecules with regard to the surface normal vector for the contact layers and the bulk layers, as shown in Figure S1. By following the simulation setup in Ref. 1, the surface normal of Ag-(0001) points along the $z$-direction, thus $\cos (\varphi)=-1$ and $\cos (\varphi)=+1$ correspond to an $\mathrm{O}-\mathrm{H}$ bond directed away from and toward the silver exposed surface, respectively.

To study the templating effect of $\mathrm{AgI}$ at $\boldsymbol{D}_{\mathbf{C N C}}$ and at $\boldsymbol{D}_{\mathbf{C N C}}+\mathrm{NaCl}$ electrolyte, we compared the ice nucleation rates among different $\mathrm{AgI}$ surfaces $(\delta=0 \%$ and $\delta=1.59 \%)$. The AgI surface with $\delta=1.59 \%$ had lattice parameters of $\mathrm{a}=4.592 \AA$ and $\mathrm{c}=7.510 \AA$. When $\delta=$ $0 \%$, the lattice constants were $\mathrm{a}=4.518 \AA$ and $\mathrm{c}=7.510 \AA$, and the AgI slab contained nine layers with $16 \mathrm{Ag}^{+}$and nine layers with $16 \mathrm{I}^{-}$. The slab width was $30.934 \AA$, and the lateral dimensions of the simulation cell were $\mathrm{Lx}=18.072 \AA$ and $\mathrm{Ly}=15.636 \AA$. The total length of the simulation box was $120.417 \AA$ along the $z$-direction, and the $\boldsymbol{D}_{\mathbf{C N C}}$ was changed to -15.50 $\mathrm{V} / \mathrm{nm}$ to achieve the compensating net charge condition.

For all the AgI surfaces $(\delta=0 \%$ and $\delta=1.59 \%)$, each simulation trajectory was first relaxed at $298 \mathrm{~K}$ for $2 \mathrm{~ns}$ before being cooled down to $242 \mathrm{~K}$ (a supercooling of $10 \mathrm{~K}$ ) within 2 ns. Simulations were then conducted at $242 \mathrm{~K}$ and terminated once nucleation occurred. For each surface, 10 parallel simulations with different initial velocities were performed. Then, the ice nucleation rates were calculated using the method presented by Cox et al., ${ }^{18}$ and the results are shown in Figure S2b-c. 


\section{SI Figures and Tables:}
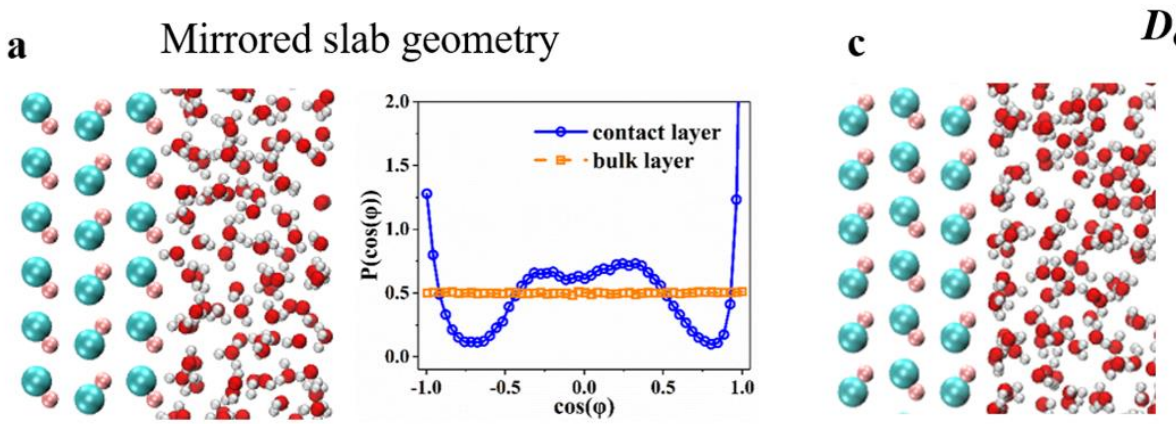

$D_{\mathrm{CNC}}$

b YB correction with large vacuum space
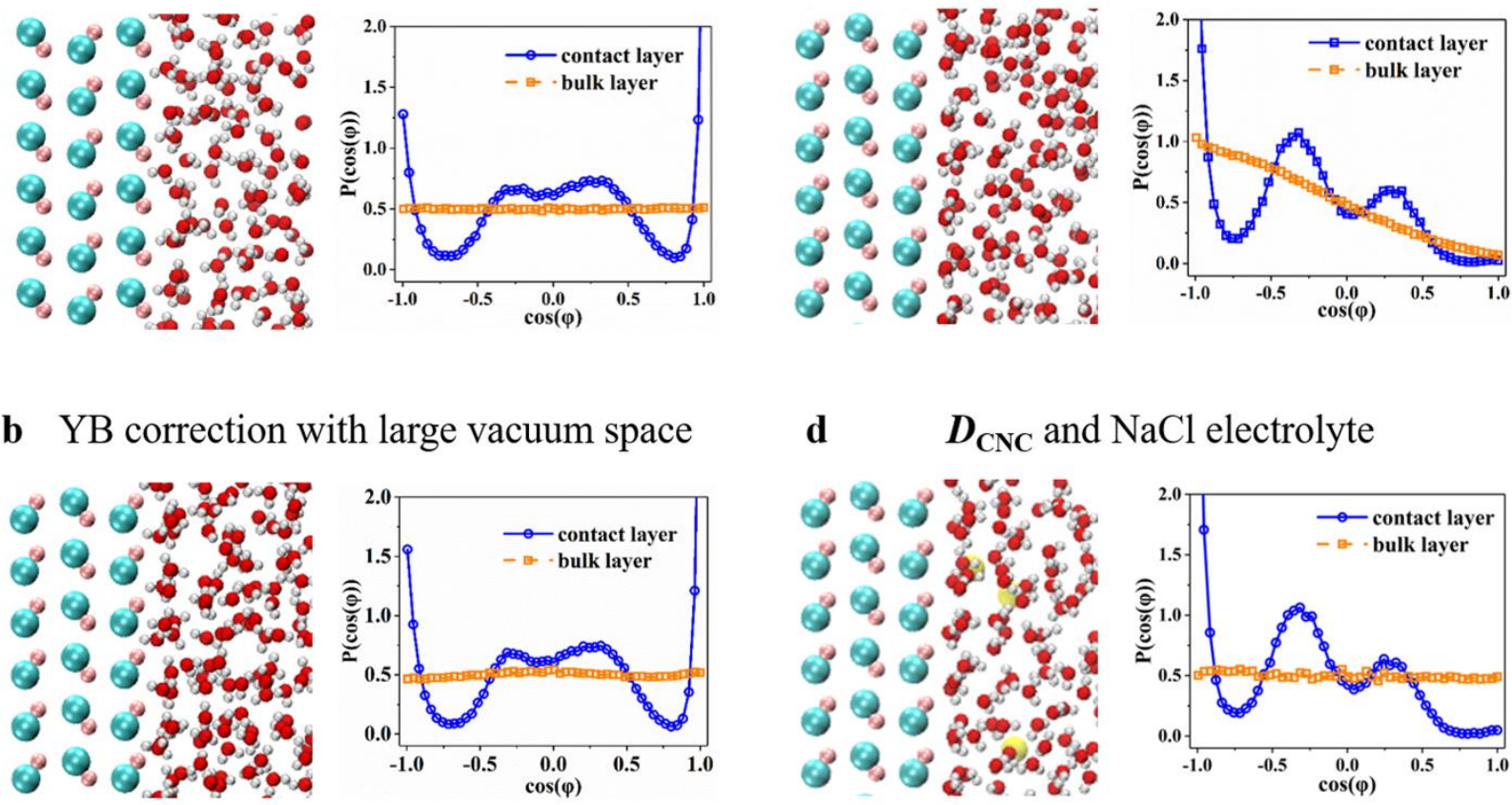

Figure S1. The orientations of water molecules at the interface. (a-d) Left panels are the closeup snapshots of the $\mathrm{Ag}-(0001) / \mathrm{H}_{2} \mathrm{O}$ interface with an immobile $\mathrm{AgI}$ crystal at $298 \mathrm{~K}$ using the mirrored slab geometry, YB correction with a volume factor of 5.0, $\boldsymbol{D}_{\mathbf{C N C}}$, and $\boldsymbol{D}_{\mathbf{C N C}}+\mathrm{NaCl}$ electrolyte, respectively. Right panels are the corresponding $\mathrm{O}-\mathrm{H}$ bonds orientation distributions with respect to the surface normal vector of water molecules in the contact layer (blue circles) and bulk layer (orange squares). In (a and b), a significant proportion of water molecules in the contact layer direct $\mathrm{O}-\mathrm{H}$ bonds toward the positively charged Ag-(0001) surface. In contrast, in the $\boldsymbol{D}_{\mathbf{C N C}}$ and $\boldsymbol{D}_{\mathbf{C N C}}+\mathrm{NaCl}$ electrolyte setups, no O-H bonds are directed toward the interface, as shown in (c and d). Silver ions, iodide ions, chloride ions, oxygen atoms, and hydrogen atoms are shown as pink, green, yellow, red, and white spheres, respectively. Only part of the simulation cell is shown. $\cos (\varphi)=+1$ and $\cos (\varphi)=-1$ indicate O$\mathrm{H}$ bonds directed immediately toward and away from Ag-(0001), respectively. Note that the systems with the mirrored slab geometry and YB corrections are simulated with the TIP4P/Ice 
water model, while the systems with $\boldsymbol{D}_{\mathbf{C N C}}$ are simulated with the TIP4P/2005 water model. All results are obtained over $1 \mathrm{~ns}$ to 2 ns at $298 \mathrm{~K}$.
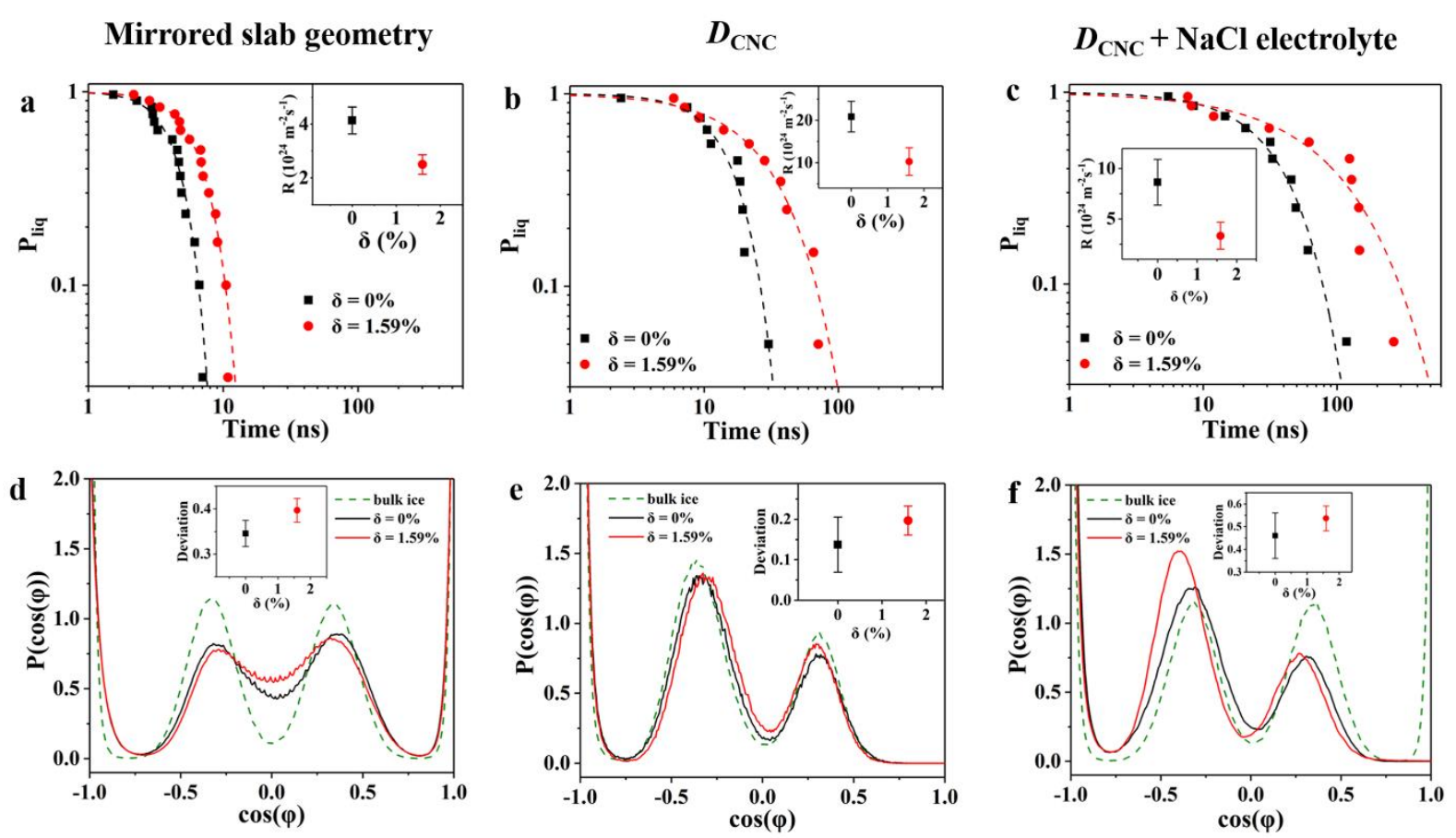

Figure S2. Ice nucleation rates on the $\beta-\operatorname{AgI}(0001)$ surfaces at different lattice and the corresponding $\mathrm{O}-\mathrm{H}$ bonds orientation distributions. Probability of liquid, $\mathrm{P}_{\text {liq, }}$, as a function of time at different values of lattice mismatch $\delta$ (a) with the mirrored slab geometry, (b) at $\boldsymbol{D}_{\mathbf{C N C}}$ and (c) at $\boldsymbol{D}_{\mathbf{C N C}}+\mathrm{NaCl}$ electrolyte, respectively. The insets show the nucleation rates as a function of $\delta$. The nucleation rates were calculated by fitting the $\mathrm{P}_{\text {liq }}$ versus time data into the exponential decay function. The error bars for the nucleation rates were computed using the Jackknife resampling technique. O-H bonds orientation distributions with respect to the surface normal vector for bulk ice and the first hydration layers at various values of lattice mismatch $\delta$ averaged from $1 \mathrm{~ns}$ to $2 \mathrm{~ns}$ of the simulation (d) with the mirrored slab geometry at $250 \mathrm{~K}$, (e) at $\boldsymbol{D}_{\mathbf{C N C}}$ at $242 \mathrm{~K}$, and (f) at $\boldsymbol{D}_{\mathbf{C N C}}+\mathrm{NaCl}$ electrolyte at $242 \mathrm{~K}$, respectively. The insets represent the deviations of the $\mathrm{O}-\mathrm{H}$ bonds distribution between the first hydration layer and bulk ice as a function of $\delta$. The error bars in the insets are the standard deviations from the 10 simulations in (e-f) and from the 15 simulations in (d). 
Table S1. Summarized LJ interaction parameters $\left(\epsilon_{\mathrm{LJ}}, \sigma_{\mathrm{LJ}}\right)$ for the simulations at $\boldsymbol{D}_{\mathbf{C N C}} \epsilon_{\mathrm{LJ}}$ is reported in $\mathrm{meV}$, and $\sigma_{\mathrm{LJ}}$ is reported in Ångstrom. '-' indicates no LJ interaction is specified between this pair of species.

\begin{tabular}{llllll}
\hline & $\mathrm{O}$ & $\mathrm{Na}+$ & $\mathrm{Cl}$ & $\mathrm{Ag}+$ & $\mathrm{I}-$ \\
\hline $\mathrm{O}$ & $(8.0310,3.1589)$ & $(8.2218,2.5134)$ & $(0.6418,4.2687)$ & $(23.7072,3.1710)$ & $(26.9725,3.3420)$ \\
$\mathrm{Na}+$ & & $(15.2598,2.2174)$ & $(14.9129,2.9051)$ & $(32.6792,2.7002)$ & $(37.1804,2.8712)$ \\
$\mathrm{Cl}-$ & & $(0.7979,4.8491)$ & $(7.4716,4.0161)$ & $(8.4994,4.1871)$ \\
$\mathrm{Ag}+$ & & & - & - \\
$\mathrm{I}-$ & & & & - \\
\hline
\end{tabular}




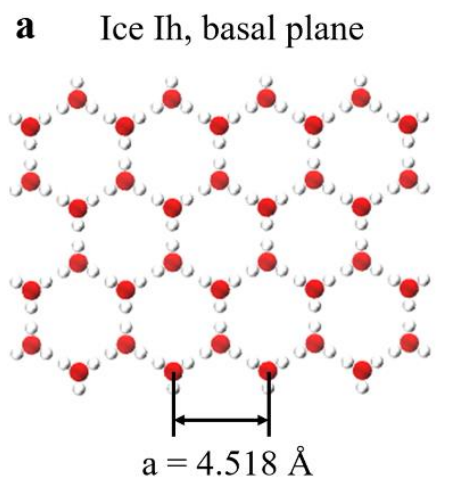

b $\quad \beta-A g I(0001)$ surface

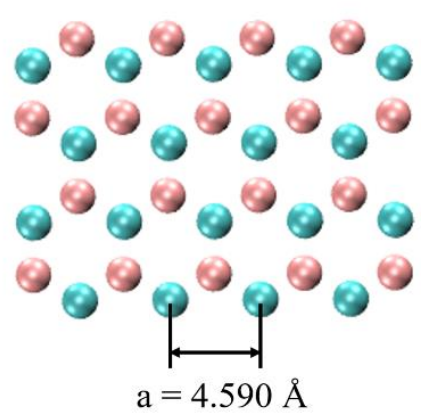

Figure S3. (a) Basal plane of ice $\mathrm{Ih}^{19}$ and (b) the (0001) surface of $\beta$-AgI seen along the $\mathrm{c}$ axis of the crystals. ${ }^{20}$ The lattice mismatch is $1.59 \%$. Oxygen atoms, hydrogen atoms, silver ions, and iodide ions are shown as red, white, pink, and green spheres, respectively. 

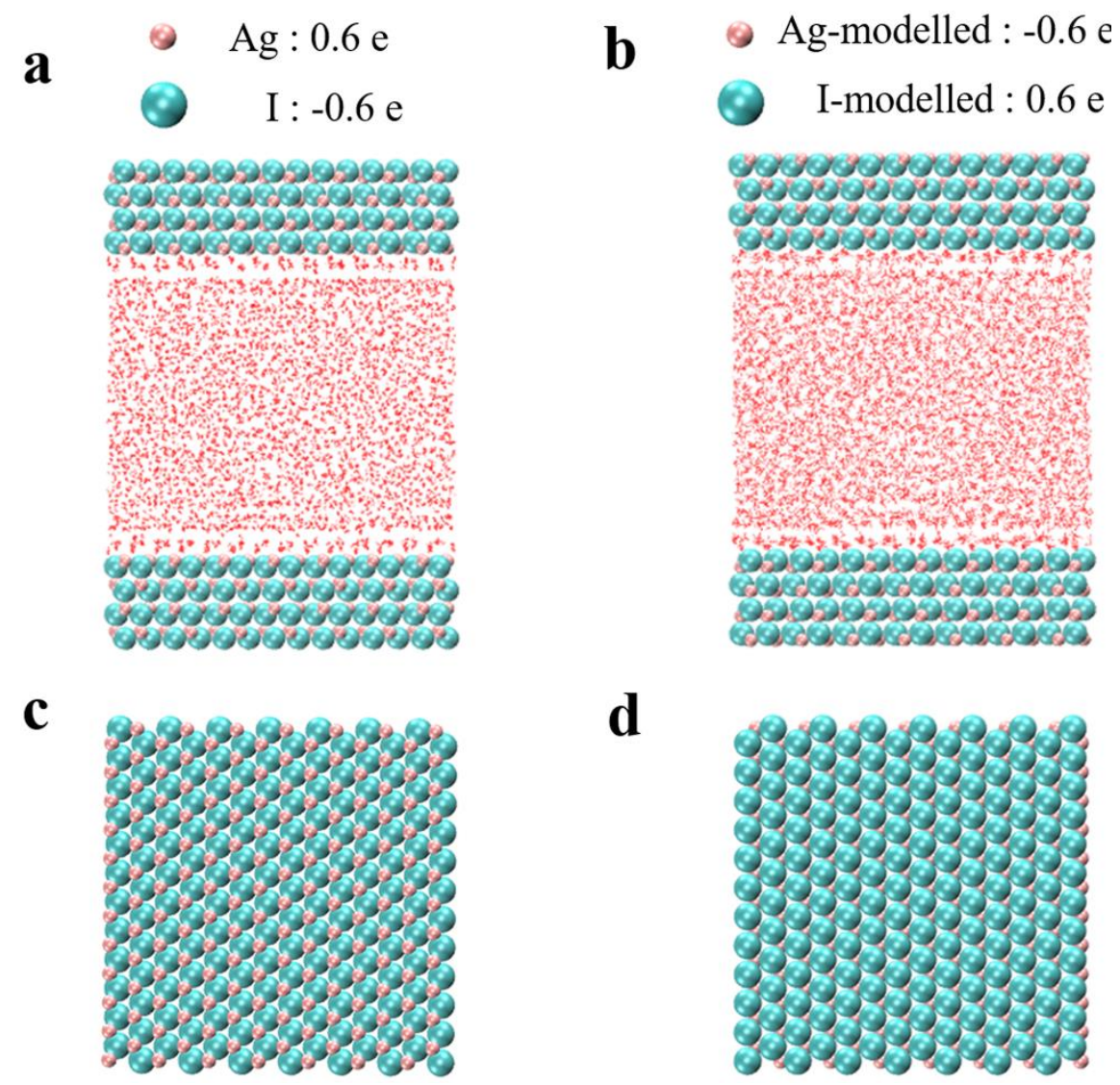

d

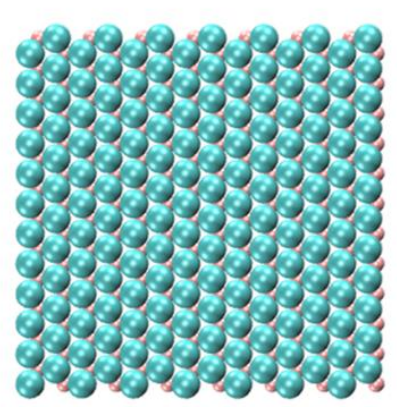

Figure S4. Simulation setups of (a) the silver exposed $\beta$-AgI(0001) surface and (b) our modelled flat surface system. Note that the charge of the iodide ions is set as $0.6 \mathrm{e}$ at the modelled flat surface, as shown in panel b. Top view snapshots of (c) the AgI surface and (d) the modelled flat surface. Silver ions, iodide ions, oxygen atoms, and hydrogen atoms are shown as pink, green, red, and white spheres, respectively. 
Table S2. Summary of the surfaces considered, the associated XY dimensions of the simulation boxes, the heights and volumes for water, the numbers of water molecules, bulk densities of liquid water, simulation runs and times, and nucleation events observed.

\begin{tabular}{|c|c|c|c|c|c|c|c|c|c|c|c|}
\hline lattice & $\begin{array}{l}\text { lattice } \\
\text { mismatch }\end{array}$ & $\begin{array}{l}\text { Exposed } \\
\text { surface }\end{array}$ & $x(\AA)$ & y $(\AA)$ & $\mathrm{z}(\AA)$ & $V\left(\AA^{3}\right)$ & $\begin{array}{l}\text { No. of } \\
\text { water }\end{array}$ & $\begin{array}{l}\text { density } \\
(\mathrm{g} / \mathrm{cm} 3)\end{array}$ & \multicolumn{2}{|c|}{ Runs } & $\begin{array}{l}\text { Nucleation } \\
\text { events }\end{array}$ \\
\hline 4.300 & $-4.83 \%$ & $\mathrm{Ag}$ & 52.15 & 51.60 & 56.96 & & & & 5 & 500 & 0 \\
\hline 4.400 & $-2.61 \%$ & $\mathrm{Ag}$ & 53.34 & 52.80 & 54.42 & & & & 15 & 80 & 15 \\
\hline 4.450 & $-1.51 \%$ & $\mathrm{Ag}$ & 53.95 & 53.40 & 53.20 & & & & 15 & 36 & 15 \\
\hline 4.518 & $0.00 \%$ & $\mathrm{Ag}$ & 54.82 & 54.22 & 51.56 & & & & 15 & 20 & 15 \\
\hline & & & & & & 153260.1 & 4610 & 0.90 & & & \\
\hline 4.590 & $1.59 \%$ & $\mathrm{Ag}$ & 55.65 & 55.08 & 50.00 & & & & 15 & 60 & 15 \\
\hline 4.590 & $1.59 \%$ & I & 55.65 & 55.08 & 50.00 & & & & 15 & 60 & 15 \\
\hline 4.700 & $4.03 \%$ & $\mathrm{Ag}$ & 56.96 & 56.35 & 47.75 & & & & 15 & 80 & 15 \\
\hline 4.800 & $6.24 \%$ & $\mathrm{Ag}$ & 58.17 & 57.60 & 45.74 & & & & 5 & 500 & 0 \\
\hline
\end{tabular}




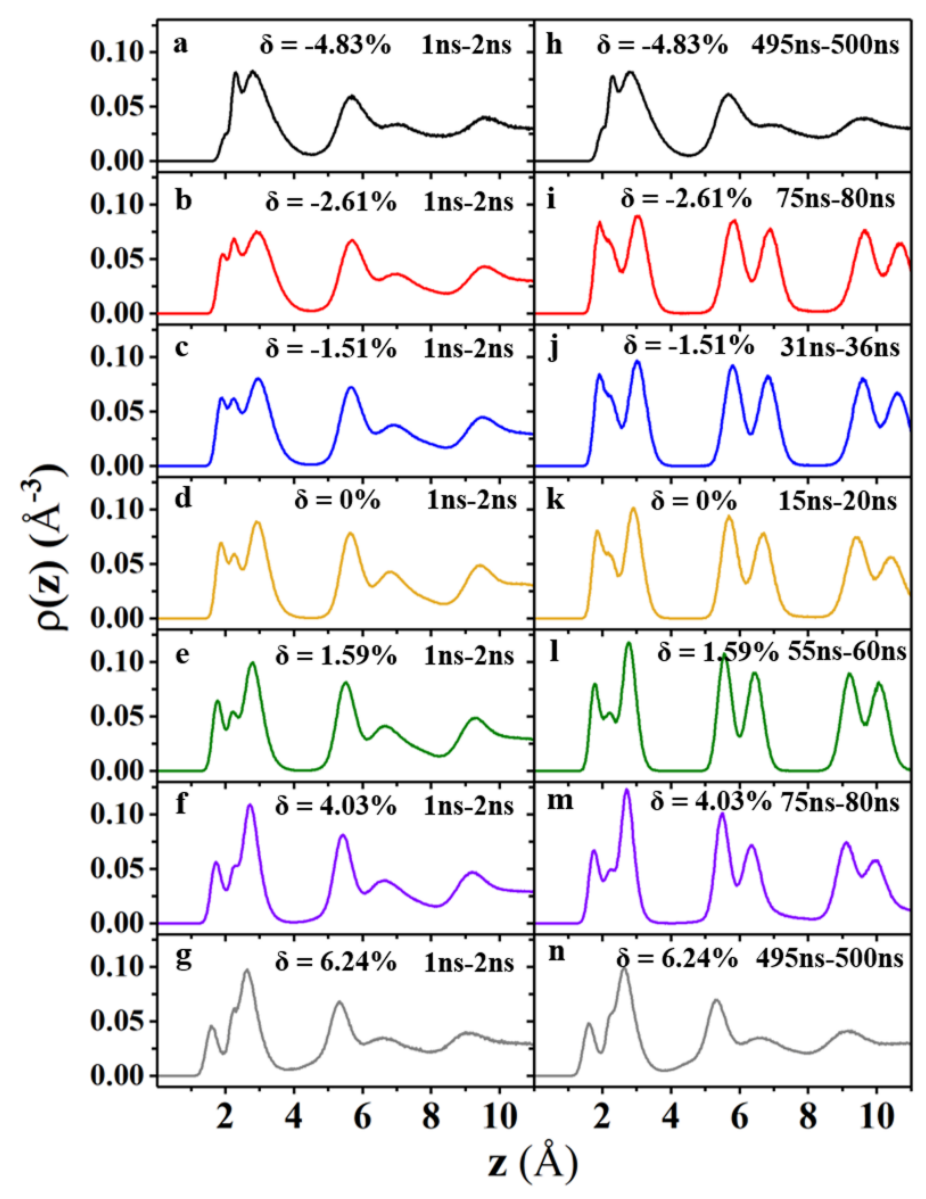

Figure S5. Number density profiles of oxygen atoms $(\rho(z))$ as a function of the distance $(z)$ from the $\beta-\operatorname{AgI}(0001)$ surfaces at $250 \mathrm{~K}$. The density profiles with (a) $\delta=-4.83 \%$, (b) $\delta=-$ $2.61 \%$, (c) $\delta=-1.51 \%$, (d) $\delta=0 \%$, (e) $\delta=1.59 \%$, (f) $\delta=4.03 \%$, and (g) $\delta=6.24 \%$ were averaged from $1 \mathrm{~ns}$ to $2 \mathrm{~ns}$, while the density profiles with (h) $\delta=-4.83 \%$, (i) $\delta=-2.61 \%$, (j) $\delta$ $=-1.51 \%,(\mathrm{k}) \delta=0 \%,(\mathrm{l}) \delta=1.59 \%,(\mathrm{~m}) \delta=4.03 \%$, and $(\mathrm{n}) \delta=6.24 \%$ were averaged from $495 \mathrm{~ns}$ to $500 \mathrm{~ns}, 75 \mathrm{~ns}$ to $80 \mathrm{~ns}, 31 \mathrm{~ns}$ to $36 \mathrm{~ns}, 15 \mathrm{~ns}$ to $20 \mathrm{~ns}, 55 \mathrm{~ns}$ to $60 \mathrm{~ns}, 75 \mathrm{~ns}$ to $80 \mathrm{~ns}$, and $495 \mathrm{~ns}$ to $500 \mathrm{~ns}$, respectively. On the distance scale, zero coincides with the position of the center of the outermost ion of the face. 

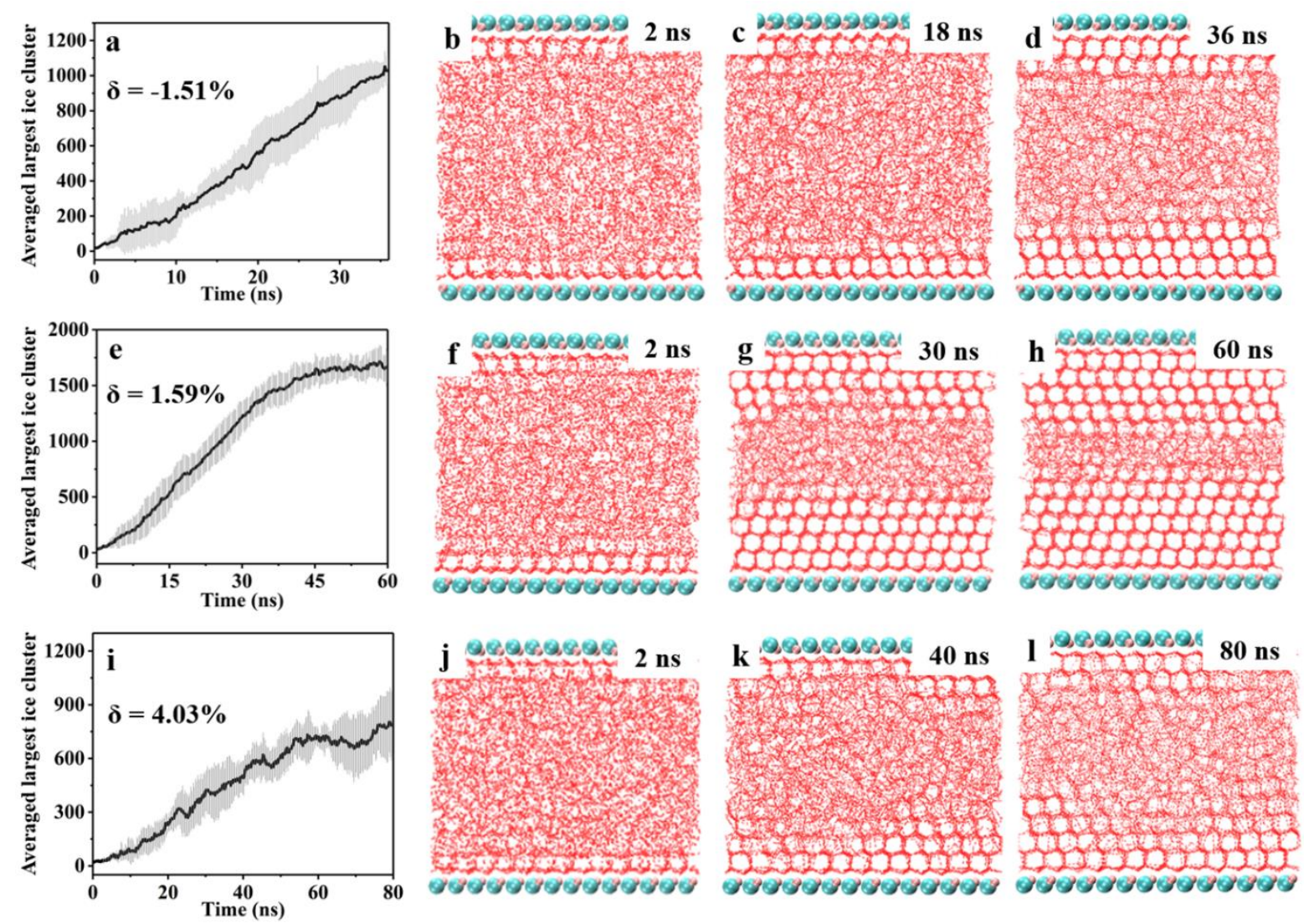

Figure S6. The averaged largest ice cluster size as a function of time and the corresponding snapshots for ice nucleation on the surfaces with $\delta=-1.51 \%, \delta=1.59 \%$, and $\delta=4.03 \%$. The averaged largest ice cluster size vs. time plot of ice nucleation on the AgI surface with (a) $\delta=$ $-1.51 \%$, (e) $\delta=1.59 \%$, and (i) $\delta=4.03 \%$ for five representative systems averaged over every configuration in which nucleation was observed. The shaded bars in panels (a), (e), and (i) correspond to the standard deviations. (b), (c), (d), (f), (g), (h), (j), (k), and (l) display the snapshots of ice growth for one representative trajectory on the AgI surface with $\delta=-1.51 \%$ at $2 \mathrm{~ns}, \delta=-1.51 \%$ at $18 \mathrm{~ns}, \delta=-1.51 \%$ at $36 \mathrm{~ns}, \delta=1.59 \%$ at $2 \mathrm{~ns}, \delta=1.59 \%$ at $30 \mathrm{~ns}, \delta=1.59 \%$ at $60 \mathrm{~ns}, \delta=4.03 \%$ at $2 \mathrm{~ns}, \delta=4.03 \%$ at $40 \mathrm{~ns}$, and $\delta=4.03 \%$ at $80 \mathrm{~ns}$, respectively. Only the first layer of each AgI slab is shown. Silver and iodide ions are shown as pink and green spheres, respectively. Water molecules are shown as hydrogen bonds in red dash lines. 

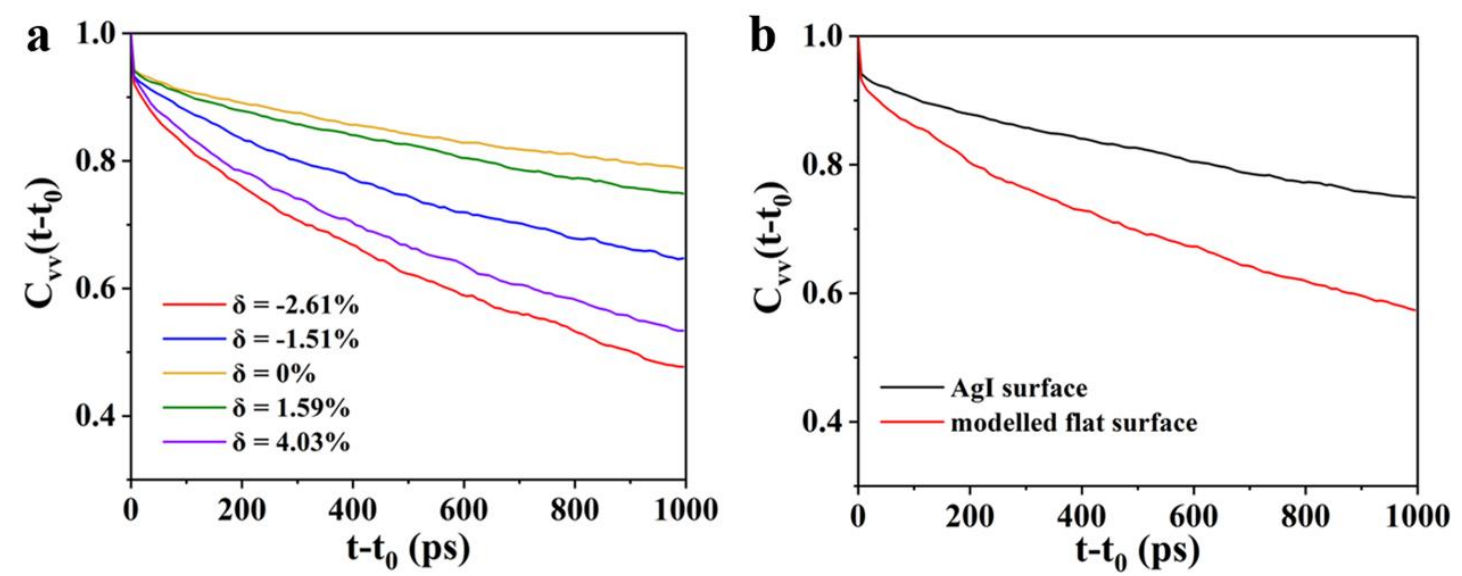

Figure S7. Dipole orientation correlation of the first hydration layer over the time range 1-2 ns at $250 \mathrm{~K}$. (a) Water dipole orientation correlation function at various values of lattice mismatch $\delta$. (b) Water dipole orientation correlation function on the AgI surface and the modelled flat surface. The dipole orientation correlation function of water molecules is defined as $C_{v v}\left(t-t_{0}\right)=\left\langle\delta \vec{v}_{i}(t) \cdot \delta \vec{v}_{i}\left(t_{0}\right) \cdot \psi_{i}\left(t_{0}\right)\right\rangle$, where $\delta \vec{v}_{i}(t)$ is the unit dipole vector of water molecule $i$ at time $t, t_{0}$ is a reference time, $\langle\cdot\rangle$ symbolises the normalised ensemble average and $\psi_{i}\left(t_{0}\right)$ is either 1 if water molecule is within the considered layer at $t_{0}$ or else 0 . The slower decay of $C_{v v}\left(t-t_{0}\right)$ corresponds to the more stable hydrogen bond network. ${ }^{21-22}$ 

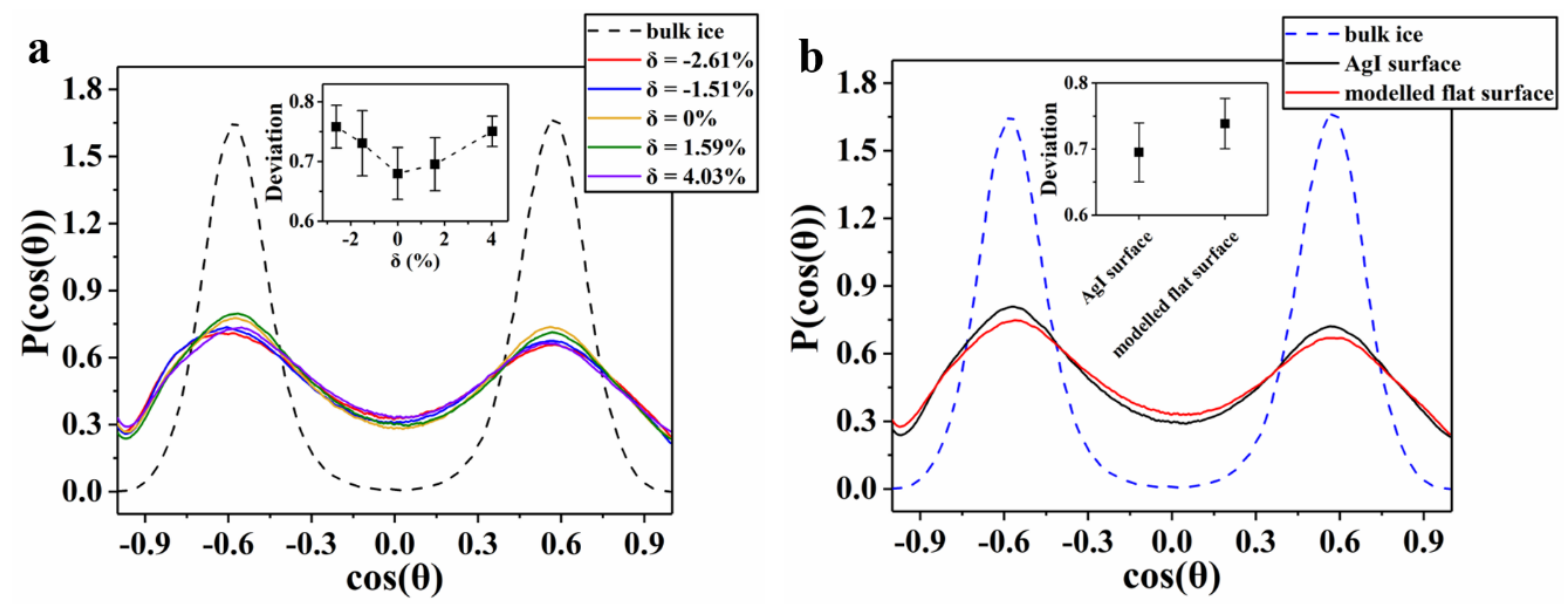

Figure S8. Water orientation analyses of the second hydration layers on the $\beta-\operatorname{AgI}(0001)$ surfaces. (a) Water dipole angle distributions with respect to the surface normal vector for the second hydration layers at various values of lattice mismatch $\delta$ averaged from 1 ns to 2 ns of the simulation at $250 \mathrm{~K}$ and bulk ice. (b) Water dipole angle distributions with respect to the surface normal vector for the second hydration layer on the AgI surface and the modelled flat surface averaged from $1 \mathrm{~ns}$ to $2 \mathrm{~ns}$ of the simulation at $250 \mathrm{~K}$ and bulk ice. The insets represent the deviations of the water dipole distribution between the second hydration layers and bulk ice. The error bars in the insets are the standard deviations from the 15 simulations. 


\section{References}

(1) Sayer, T.; Cox, S. J. Stabilization of AgI's Polar Surfaces by the Aqueous Environment, and Its Implications for Ice Formation. Phys. Chem. Chem. Phys. 2019, 21, 14546-14555.

(2) Spohr, E. Effect of Electrostatic Boundary Conditions and System Size on the Interfacial Properties of Water and Aqueous Solutions. J. Chem. Phys. 1997, 107, 6342-6348.

(3) Yeh, I.-C.; Berkowitz, M. L. Ewald Summation for Systems with Slab Geometry. J. Chem. Phys. 1999, 111, 3155-3162.

(4) Abdelmonem, A.; Backus, E. H. G.; Hoffmann, N.; Sánchez, M. A.; Cyran, J. D.; Kiselev, A.; Bonn, M. Surface-Charge-Induced Orientation of Interfacial Water Suppresses Heterogeneous Ice Nucleation on $\alpha$-Alumina (0001). Atmos. Chem. Phys. 2017, 17, 7827-7837.

(5) Soni, A.; Patey, G. N. Why $\alpha$-Alumina Is an Effective Ice Nucleus. J. Phys. Chem. C 2019, 123, 26424-26431.

(6) Ranea, V. A.; Schneider, W. F.; Carmichael, I. DFT Characterization of Coverage Dependent Molecular Water Adsorption Modes on $\alpha-\mathrm{Al}_{2} \mathrm{O}_{3}(0001)$. Surf. Sci. 2008, 602, 268 275.

(7) Kondo, T.; Ito, T. Flipping Water Molecules at Insulator/Solution Interface Using an Externally Applied Weak Electric Field. Appl. Phys. Lett. 2014, 104, 101601.

(8) Liao, B.; Qiu, L.; Wang, D.; Bao, W.; Wei, Y.; Wang, Y. The Behaviour of Water on the Surface of Kaolinite with an Oscillating Electric Field. RSC Adv. 2019, 9, 21793-21803.

(9) Fraux, G.; Doye, J. P. K. Note: Heterogeneous Ice Nucleation on Silver-Iodide-Like Surfaces. J. Chem. Phys. 2014, 141, 216101.

(10) Abascal, J. L. F.; Vega, C. A General Purpose Model for the Condensed Phases of Water: TIP4P/2005. J. Chem. Phys. 2005, 123, 234505.

(11) Benavides, A. L.; Portillo, M. A.; Chamorro, V. C.; Espinosa, J. R.; Abascal, J. L. F.; Vega, C. A Potential Model for Sodium Chloride Solutions Based on the TIP4P/2005 Water Model. J. Chem. Phys. 2017, 147, 104501.

(12) Hale, B. N.; Kiefer, J. Studies of $\mathrm{H}_{2} \mathrm{O}$ on $\beta$-AgI Surfaces: An Effective Pair Potential Model. J. Chem. Phys. 1980, 73, 923-933.

(13) Zielke, S. A.; Bertram, A. K.; Patey, G. N. A Molecular Mechanism of Ice Nucleation on Model AgI Surfaces. J. Phys. Chem. B 2015, 119, 9049-9055.

(14) Plimpton, S. Fast Parallel Algorithms for Short-Range Molecular Dynamics. J. Comput. Phys. 1995, 117, 1-19.

(15) Cox, S. J.; Sprik, M. Finite Field Formalism for Bulk Electrolyte Solutions. J. Chem. Phys. 2019, 151, 064506.

(16) Andersen, H. C. Rattle: A "Velocity" Version of the Shake Algorithm for Molecular Dynamics Calculations. J. Comput. Phys. 1983, 52, 24-34.

(17) Hockney, R. W.; Eastwood, J. W. Computer Simulation Using Particles. CRC Press: Boca Raton, 1988.

(18) Cox, S. J.; Kathmann, S. M.; Slater, B.; Michaelides, A. Molecular Simulations of Heterogeneous Ice Nucleation. I. Controlling Ice Nucleation through Surface Hydrophilicity. J. Chem. Phys. 2015, 142, 184704.

(19) Rottger, K.; Endriss, A.; Ihringer, J.; Doyle, S.; Kuhs, W. F. Lattice Constants and Thermal Expansion of $\mathrm{H}_{2} \mathrm{O}$ and $\mathrm{D}_{2} \mathrm{O}$ Ice Ih between 10 and 265 K. Acta Crystallogr., Sect. B 1994, 50, 644-648.

(20) Bührer, W.; Nicklow, R. M.; Brüesch, P. Lattice Dynamics of $\beta$-(Silver Iodide) by Neutron Scattering. Phys. Rev. B 1978, 17, 3362-3370.

(21) Li, C.; Gao, X.; Li, Z. Roles of Surface Energy and Temperature in Heterogeneous Ice Nucleation. J. Phys. Chem. C 2017, 121, 11552-11559. 
(22) Prerna; Goswami, R.; Metya, A. K.; Shevkunov, S. V.; Singh, J. K. Study of Ice Nucleation on Silver Iodide Surface with Defects. Mol. Phys. 2019, 117, 3651-3663. 